\title{
TIME-RESOLVED RESONANCE RAMAN SPECTROSCOPY OF PHOTOCHEMICAL REACTIVE INTERMEDIATES: RADICAL CATION OF FLUORENE AND TRIPLET STATE OF FLUORENE, DIBENZOFURAN AND DIBENZOTHIOPHEN
}

\author{
G. BUNTINX \\ Laboratoire de Spectrochimie Infrarouge et Raman, CNRS, \\ USTL, 59655 Villeneuve d'Ascq (France) \\ O. POIZAT \\ Laboratoire de Spectrochimie Infrarouge et Raman, CNRS, \\ 2 rue Henri Dunant, 94320 Thiais (France)
}

(Received 15 October, 1989; in final form 5 January, 1990)

\begin{abstract}
The time-resolved Raman spectra of the first triplet state-in resonance with the $T_{n} \leftarrow T_{1}$ absorption at $370 \mathrm{~nm}$ - and of the radical cation transient-in resonance with the $R^{+.^{*}} \leftarrow R^{+\cdot}$ absorptions at $370 \mathrm{~nm}$ and in the 560-600 $\mathrm{nm}$ range-are reported for fluorene. The triplet state Raman spectra of dibenzofuran and dibenzothiophen are also given. The vibrational assignments, resonance Raman activity and intensity enhancement effects are studied. On this basis, the structures and electronic configurations of the triplet state and radical cation transients and the nature of the resonant $T_{n} \leftarrow T_{1}$ and $R^{+\cdot^{*}} \leftarrow R^{+\cdot}$ transitions are discussed. It turns out from this investigation that the title molecules present close analogies with biphenyl. The insertion of a methylene group or a heteroatom does not disturb markedly the electronic properties of the ground state, the first triplet state and the radical cation transient of biphenyl.
\end{abstract}

\section{INTRODUCTION}

Reactive intermediates such as excited singlet and triplet states, ions, and radicals, play a determinant role in most chemical and photochemical processes. In this regard, the spectroscopic investigation of these transient species is essential for understanding the molecular reactivity and a considerable work has been done by using time-resolved methods such as absorption and emission spectroscopies, photoconductivity and chemically induced dynamic nuclear polarization (CIDNP) or ESR spectroscopy. In particular, a great deal of chemical information can be obtained from transient absorption. Nonetheless, in condensed phases, absorption bands are generally broad and diffuse with limited inherent structural information. In contrast, resonance Raman spectroscopy is a particularly efficient technique to provide detailed structural information and excellent discrimination between species which present broad and overlapping absorption bands. 
The Raman analysis of short-lived intermediate species has been made possible owing to the pioneering development by Bridoux and Delhaye ${ }^{1}$ of the principle of multichannel analysis for Raman spectroscopy, and the considerable improvement in sensitivity and temporal resolution that has been realized in the last years by Bridoux et al. ${ }^{2,3}$ The first generation of multichannel Raman spectrometer used photographic plates as detective materials and had a comparatively low sensitivity. A second generation of instruments relied on image intensifiers and low light level television cameras. ${ }^{1 \mathrm{a}, 1 \mathrm{~b}}$ These detectors were characterized by high sensitivity but low stability, distortion of the image and limited resolution and dynamic range. Nevertheless, they were employed for obtaining the first vibrational spectra in the nanosecond, then the picosecond, time scales. ${ }^{2}$ A third generation of multichannel spectrometers, using intensified photodiode arrays as detectors, has been recently developed. ${ }^{3}$ With the advantage of being gatable in the $10 \mathrm{~ns}$ range, they present high stability, dynamic and sensitivity characteristics. Since the first observation of a species in an excited state-the lowest triplet state of paraterphenyl-by Wilbrandt et al. ${ }^{4}$ the application of this technique to the vibrational detection of reactive intermediates has been largely adopted. ${ }^{5}$

Recently, we have developed an extensive resonance Raman investigation of photochemical transients of aromatic molecules by using a nanosecond pump/probe excitation system. By this method, the transient species is generated with a first laser excitation (pump pulse) and its Raman spectrum is induced by a second laser excitation (probe pulse) which is retarded from the former by an adjustable time delay. In previous works, we have investigated the lowest triplet state $\left(T_{1}\right)$ and the radical cation state $\left(R^{+}\right)$of aromatic amines ${ }^{6-9}$ and of various derivatives of biphenyl. ${ }^{10,11}$ The present paper deals with the photoexcited triplet state of fluorene, dibenzofuran and dibenzothiophen (Figure 1) and the radical cation of fluorene.

Important analogies have been found between fluorene and biphenyl: both species are characterized in the triplet state by a strong $T_{n} \leftarrow T_{1}$ absorption peaking around $370 \mathrm{~nm}^{12-15}$ and in the radical cation state by two absorptions lying in the 360-390 and $530-700 \mathrm{~nm}$ regions, respectively. ${ }^{16,17}$ Molecular orbital calculations ${ }^{18}$ have suggested that the $T_{1}$ states of biphenyl $\left({ }^{3} B_{1 u}\right)$ and of fluorene $\left({ }^{3} B_{1}\right)$ result mainly from the LUMO $\leftarrow$ HOMO $\left(\pi \pi^{*}\right)$ excitation configuration and have comparable

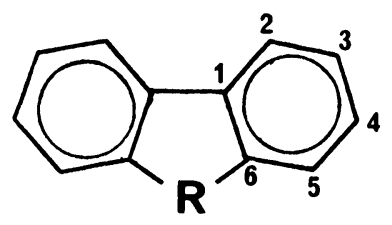

$\mathrm{R} \begin{cases}\mathrm{CH}_{2} & \text { Fluorene } \\ 0 & \text { Dibenzofurane } \\ \mathrm{S} & \text { Dibenzothiophen }\end{cases}$
Figure 1 Representation of the molecules studied and numbering of the atoms. 
orbital natures. Electron paramagnetic resonance results ${ }^{19}$ have indicated that the $T_{1}$ states of fluorene, dibenzofuran and dibenzothiophen are very similar to the $T_{1}$ state of biphenyl and that the methylene group and the heteroatoms have no significant contribution to the $T_{1} \leftarrow S_{0}$ excitation. On the other hand, very detailed information on the triplet state and radical cation species has been obtained for biphenyl from time-resolved resonance Raman studies ${ }^{5,10,11}$ The analysis of the changes in wavenumber of the Raman bands with respect to the ground state values has clearly shown that both the $T_{1}$ and $R^{+\cdot}$ structures present a quinoidal distortion with a notable increase of the inter-ring bond order. In addition, the specific resonance enhancements of the band intensities have appeared very typical of the distortions of the $T_{1}$ and $R^{+\cdot}$ structures in the resonant upper electronic states, $T_{n}$ and $\left(R^{+}\right)^{*}$, respectively, and precise information concerning the orbital origins of the $T_{n} \leftarrow T_{1}$ absorption at $370 \mathrm{~nm}$ and of the $\left(R^{+\cdot}\right)^{*} \leftarrow R^{+}$absorptions at 370 and $690 \mathrm{~nm}$ could be established, in good agreement with predictions from simple molecular orbital considerations.

Here, the resonance Raman band wavenumbers and intensities of the triplet state and radical cation of fluorene are discussed in parallel with the analysis made for biphenyl. ${ }^{11}$ The triplet state spectra of dibenzofuran and of dibenzothiopen are also studied. These results are compared to the time-resolved resonance CARS spectra and analysis reported recently for $T_{1}$ fluorene. ${ }^{20}$

\section{EXPERIMENTAL}

Fluorene, dibenzofuran and dibenzothiophen were sublimated in vacuo prior to each measurement. In particular, the highly fluorescent species fluorenone, which is always present as a yellow impurity in fluorene, had to be carefully eliminated. Acetonitrile, methanol and cyclohexane (Prolabo) were used as received. Samples were contained in static $10 \times 10 \mathrm{~mm}^{2}$ quartz cells.

A single $10 \mathrm{~Hz}$ Q-switched Nd: Yag laser (Quantel YG581C) was used for the pump and the probe pulse excitations, which were temporally delayed by an optical delay line $(\sim 30 \mathrm{~ns})$. The fourth harmonic $(266 \mathrm{~nm}, 12 \mathrm{~ns}, 1.5 \mathrm{~mJ})$ was employed as pump beam. Transient species were probed at $370 \mathrm{~nm}$ and at $625 \mathrm{~nm}$. Excitation at $370 \mathrm{~nm}(8 \mathrm{~ns}, 1.5 \mathrm{~mJ})$ was achieved by pumping rhodamine 610 in a dye laser (Quantel TDL50) with the second harmonic $(532 \mathrm{~nm}$ ) of the Yag laser, then mixing the output with the first harmonic $(1064 \mathrm{~nm})$. Probe excitation at $625 \mathrm{~nm}(8 \mathrm{~ns}$, $5 \mathrm{~mJ}$ ) was obtained directly by pumping DCM with the $532 \mathrm{~nm}$ output of the Yag laser.

Raman emission was collected at $90^{\circ}$ to the incident excitation and focussed onto the entrance slit of a home-made single-monochromator multichannel spectrometer which has been already described. ${ }^{10}$ Two easily interchangeable gratings appropriate to the 370 and $625 \mathrm{~nm}$ probe wavelengths were used, and a gated intensified photodiode array (DILOR) was employed as detector (gate $20 \mathrm{~ns} \mathrm{FWHM,} \mathrm{jitter} \mathrm{less}$ than $2 \mathrm{~ns}$ ). Spectral resolutions of 8 and $4 \mathrm{~cm}^{-1}$ and analyzed spectral fields of about 1600 and $800 \mathrm{~cm}^{-1}$ were achieved at 370 and $625 \mathrm{~nm}$, respectively. For each sample a 
spectrum was recorded with both pump and probe beams and a second spectrum with probe only: the transient spectrum was thus obtained by subtracting these two spectra. Accumulations over 500 to 2000 laser pulses were performed in order to improve the signal-to-noise ratio.

\section{RESULTS}

The time-resolved resonance Raman spectra, excited at 370 and $625 \mathrm{~nm}$, of $10^{-3} \mathrm{M}$ solutions of fluorene, dibenzofuran and dibenzothiophen in cyclohexane, acetonitrile and methanol were recorded between 200 and $1900 \mathrm{~cm}^{-1}$. A $266 \mathrm{~nm}$ pump wavelength was employed with a $30 \mathrm{~ns}$ time delay between pump and probe pulses. In the case of fluorene, the $T_{n} \leftarrow T_{1}$ extinction coefficient $\epsilon$ at $370 \mathrm{~nm}$, estimated from the transient absorption spectra reported in ref. 12 and 15 is $\sim 10^{4} \mathrm{M}^{-1} \mathrm{~cm}^{-1}$ and the quantum fluorescence yield $\phi_{F}$ is $0.80 .{ }^{21}$ These values are much less favourable to the triplet state detection than those observed for biphenyl $\left(\epsilon_{T_{n} \leftarrow T_{1}}=3.5 \times 10^{4} \mathrm{M}^{-1}\right.$ $\mathrm{cm}^{-122}$ and $\phi_{F}=0.17 .{ }^{21}$ As a matter of fact, the experimental signal-to-noise ratio of the Raman spectra were much lower for $T_{1}$ fluorene than for $T_{1}$ biphenyl. ${ }^{10,11}$ Considering the radical cation species, a $R^{+.^{*}} \leftarrow R^{+\cdot}$ absorption strength of $\sim 10^{4} \mathrm{M}^{-1} \mathrm{~cm}^{-1}$ at $625 \mathrm{~nm}$ is estimated from the transient absorption spectrum reported for fluorene ${ }^{+\cdot}$ in ref. 16.

Spectra recorded with probe pulses only show exclusively the solvent Raman peaks, indicating that the non-resonant ground state spectrum is not detectable. Spectra acquired with pump and probe pulses exhibit new Raman signals which depend on the solvent and on the probe wavelength and which are due to transient species. Figure 2 shows the ground state Raman spectra of the three title compounds, recorded from pure powder samples. Figure 3 displays the transient resonance Raman spectra obtained for fluorene in methanol and in acetontrile solvents and Figure 4 shows the transient resonance Raman spectra observed for dibenzofuran and dibenzothiophen in methanol solvent.

Consider first the fluorene derivative. In methanol (Figure 3A) and in cyclohexane, comparable strongly enhanced spectra are excited at $370 \mathrm{~nm}$ whereas no transient spectrum is observed for probe excitation at $625 \mathrm{~nm}$. This resonance effect is conform to that expected for the triplet state species which has only one absorption band in the $370 \mathrm{~nm}$ region. As shown in Figure 5, the resonance CARS spectrum reported by Matsunuma et al. for $T_{1}$ fluorene ${ }^{20}$ and the resonance Raman spectrum have five components in common, indicated by dashed lines in the figure. However, the Raman spectrum is of much better quality and exhibits eight new peaks at 1868 , $1778,1374,1222,965,813,747$ and $408 \mathrm{~cm}^{-1}$ which are ascribable to the triplet state. Two additional weak peaks at 1616 and $729 \mathrm{~cm}^{-1}$ (indicated with the symbol $\times$ in Figures 3 and 5) are observed in methanol but not in cyclohexane. They correspond to strong bands of the transient spectrum obtained in acetonitrile (Figure 3B) and can be assigned to the radical cation species (see following paragraph).

In acetonitrile, new transient spectra with different resonance effects are observed at 370 and $625 \mathrm{~nm}$ (Figures 3B and 3C, respectively). These spectra are ascribed to 


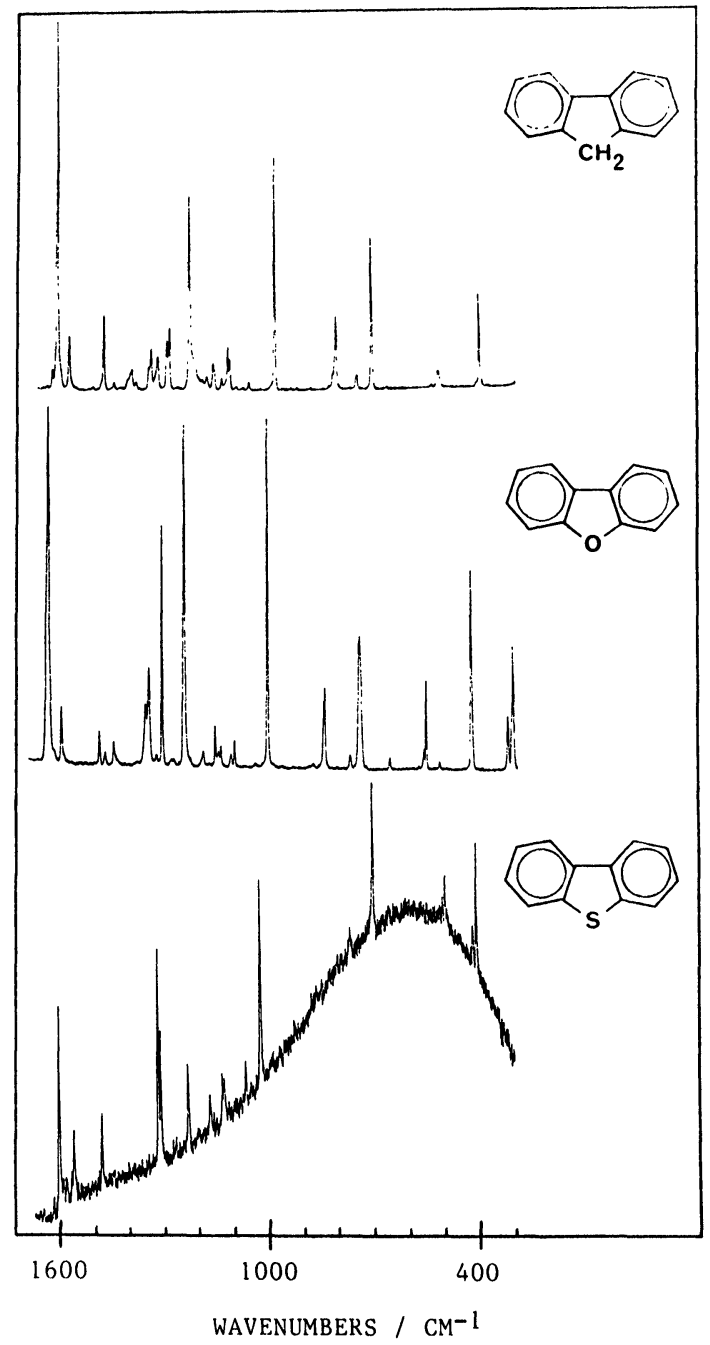

Figure 2 Raman spectra $\left(1700-300 \mathrm{~cm}^{-1}\right)$ of fluorene, dibenzofuran and dibenzothiophen in the ground state $S_{0}$ (pure powder samples). CW excitation at $647.1 \mathrm{~nm}, 50 \mathrm{~mW}$.

the fluorene radical cation by analogy with the biphenyl results. ${ }^{11}$ This assignment is in agreement wih the report by Delcourt and Rossi ${ }^{16}$ of rapid photoionization of fluorene in $\mathrm{CH}_{3} \mathrm{CN}$ solution upon $\mathrm{UV}$ excitation. The double resonance Raman enhancement at 370 and $625 \mathrm{~nm}$ is conform to the existence of $R^{+.^{*}} \leftarrow R^{+\cdot}$ transitions in both regions. Several additional weak signals in the spectrum excited at 370 $\mathrm{nm}$, noted with an asterisk in Figure 3B, are due to the triplet state. A biphotonic ionization process seems unlikely in reason of the quasi-liner dependence of the radical cation yield (as measured by the Raman intensity) upon pump pulse power. Electron ejection may occur either by direct photoionization and electron solvation, 


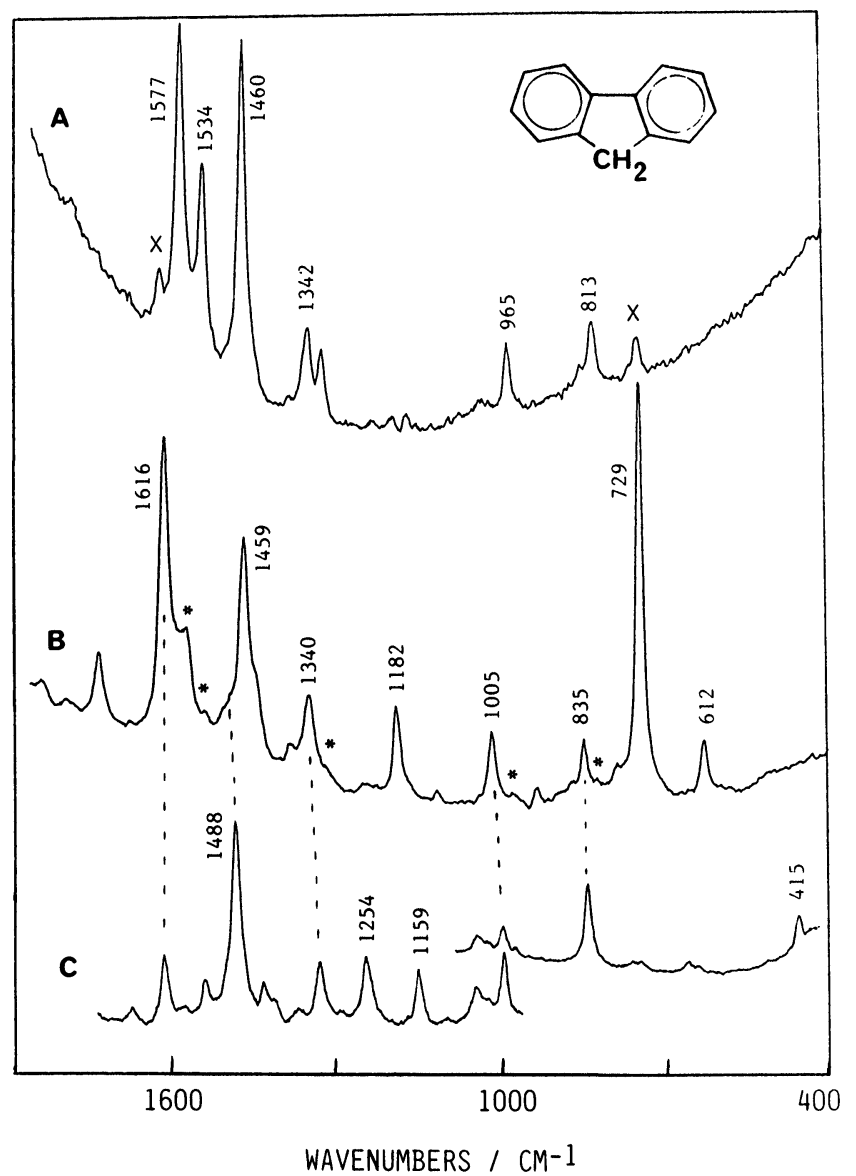

Figure 3 Original time-resolved resonance Raman spectra $\left(1800-400 \mathrm{~cm}^{-1}\right)$ of $5 \times 10^{-3} \mathrm{M}$ solutions of fluorene in (A) methanol, $\lambda_{\text {exc }}=370.0 \mathrm{~nm}$, (b) acetonitrile, $\lambda_{\text {exc }}=370.0 \mathrm{~nm},(\mathrm{C})$ acetonitrile, $\lambda_{\text {exc }}=$ $625.0 \mathrm{~nm}$. Pump excitation is at $266 \mathrm{~nm}$ and pump/probe delay is $30 \mathrm{~ns}$ in all cases. Solvent peaks have been subtracted. The principal band wavenumbers $\left(\mathrm{cm}^{-1}\right)$ are reported.

or by ionic photodissociation from a solvent-solute exciplex-type entity. The fact that photoionization is much more efficient in $\mathrm{CH}_{3} \mathrm{CN}$ than in $\mathrm{CH}_{3} \mathrm{OH}$ solutions can be accounted for by the higher solvent polarity in the first case. The absence of spectra from the ionized species in cyclohexane (only the triplet state spectrum is observed) is not surprising since this solvent is too inert to form an exciplex and to stabilize a radical cation or solvate an electron.

Finally, the transient spectra observed upon excitation at $370 \mathrm{~nm}$ for dibenzofuran and dibenzothiophen in methanol (Figure 4) and cyclohexane are comparable to those found for $T_{1}$ fluorene and, by analogy, are assigned to the triplet state species. Weak intensity spectra are excited in acetonitrile solutions which will not be discussed here in reason of their poor quality. 


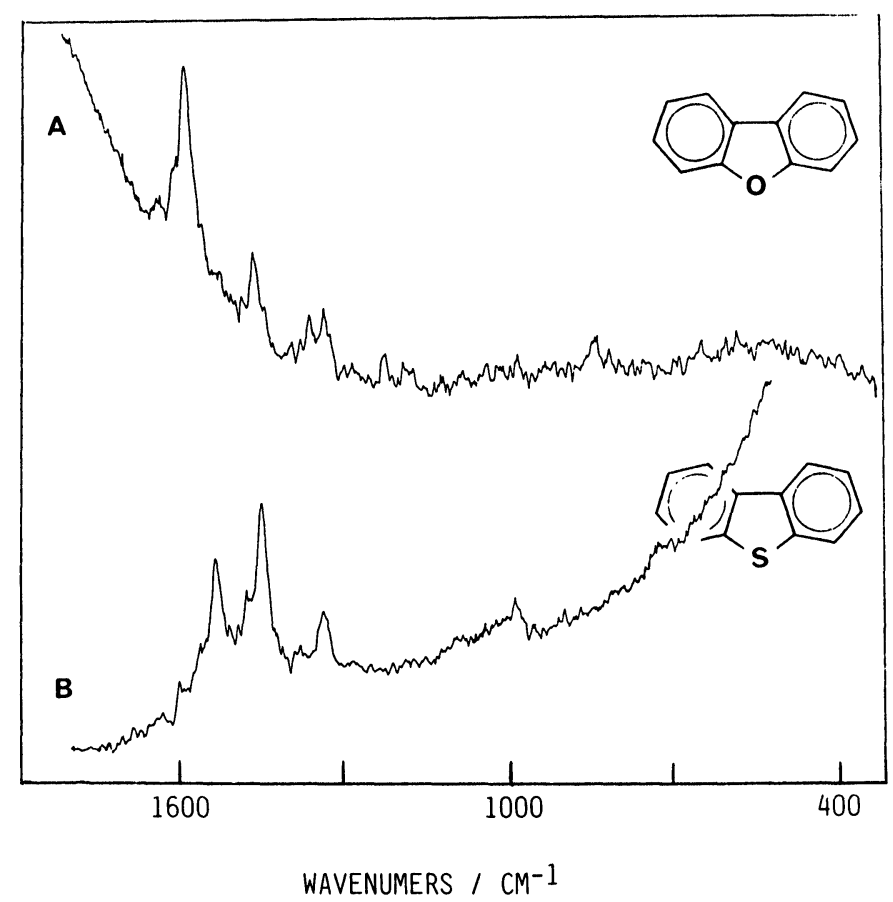

Figure 4 Original time-resolved resonance Raman spectra $\left(1750-400 \mathrm{~cm}^{-1}\right)$ of (A) dibenzofuran and (B) dibenzothiophen in methanol $\left(5 \times 10^{-3} \mathrm{M}\right.$ solutions). Pump excitation at $266 \mathrm{~nm}$, probe excitation at $370 \mathrm{~nm}$, pump/probe delay $30 \mathrm{~ns}$. Solvent peaks have been subtracted.

\section{DISCUSSION}

The experimental band wavenumber observed for the fluorene triplet state and radical cation and for the dibenzofuran and dibenzothiphen triplet state, and the proposed assignments are summarized in Tables 1 (fundamental) and 2 (overtones and combinations).

The high intensity of the $T_{n} \leftarrow T_{1}$ and $R^{+.^{*}} \leftarrow R^{+\cdot}$ absorptions in the 370 and $625 \mathrm{~nm}$ ranges and the strong enhancement of the transient Raman spectra excited in resonance with these transitions suggest that the scattering process is dominated by Franck-Condon mechanisms (A-term of the Raman scattering cross section in the Albrecht's formalism) ${ }^{23}$ Accordingly, only the totally symmetric vibrations which are disturbed in the resonant electronic transitions can be assumed to gain significant Raman intensity by resonance. Therefore, whereas the analysis of the changes in frequency in the transient states $\left(T_{1}, R^{+}\right)$with respect to the ground state must inform on the distortion of the molecule in going from $S_{0}$ to $T_{1}$ or $R^{+}$, the analysis of the resonance Raman intensities is expected to provide information on the structural distortion of the transient species $\left(T_{1}, R^{+\cdot}\right)$ in the upper electronic states $\left(T_{n}, R^{+*^{*}}\right)$, 


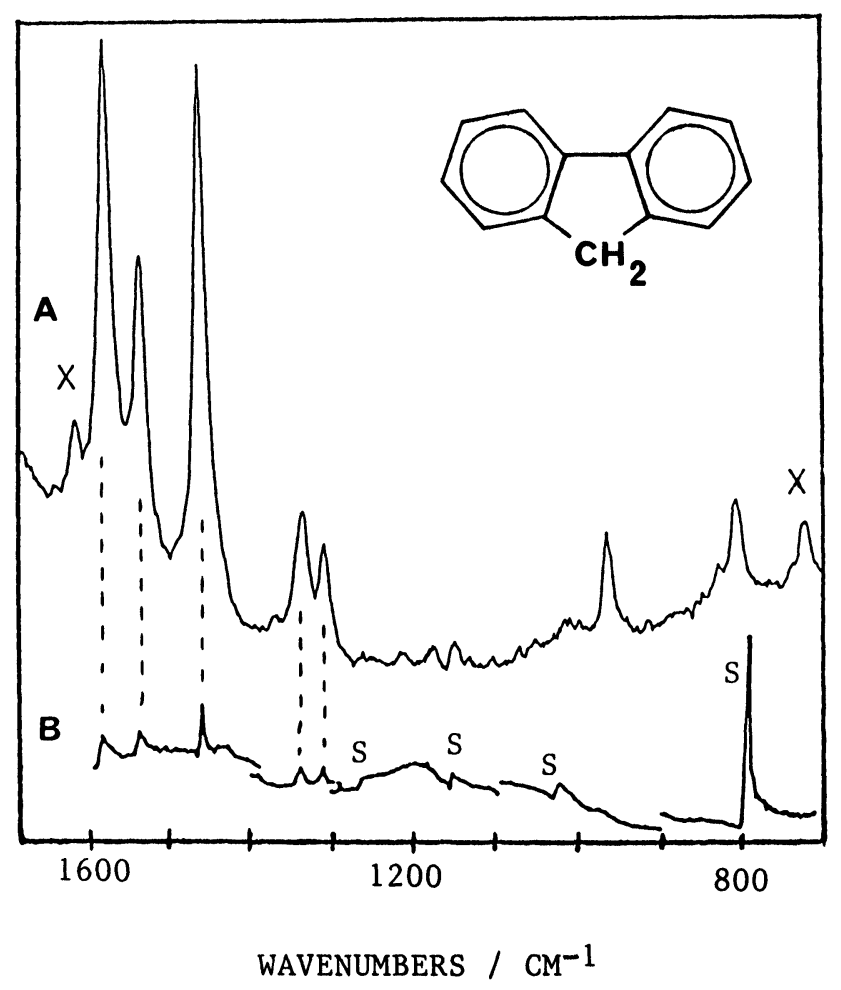

Figure 5 Comparison of the time-resolved resonance Raman (A) and CARS (from ref. 20) (B) spectra of fluorene in the $T_{1}$ state $\left(1600-700 \mathrm{~cm}^{-1}\right.$ region). The dashed lines indicate common peaks. Symbol $\times$ designates bands due to the radical cation species. Solvent peaks have been subtracted in the Raman spectrum and are pointed out by symbol $\mathrm{S}$ in the CARS spectrum.

i.e., on the nature of the $T_{n} \leftarrow T_{1}$ and $R^{+.^{*}} \leftarrow R^{+\cdot}$ absorptions. Both aspects are discussed below.

The similarity of the electronic excitation configurations (LUMO $\leftarrow$ HOMO) in $T_{1}$ biphenyl and in $T_{1}$ fluorene, dibenzofuran and dibenzothiophen ${ }^{18,19}$ suggests that in all cases the triplet structure is characterized by an increase of the inter-ring bond order (the HOMO and LUMO are mainly antibonding and bonding with respect to the inter-ring bond, respectively). Similarly, one may expect that removing a HOMO electron will also lead to a strengthening of the inter-ring CC double-bond character in biphenyl and in fluorene. Therefore, nearly planar conformations with $C_{2 v}$ symmetry can be assumed for both the triplet state and radical cation transients. In this hypothesis, sixteen totally symmetric $\left(a_{g}\right)$ vibrations are expected in the $300-1800 \mathrm{~cm}^{-1}$ region for the $C_{12} H_{8} R$ skeleton (Figure 1): thirteen ring modes and three inter-ring modes which include two stretches ( $v$ inter-ring and $v^{s}$ ring-R-ring) and one in-plane bend ( $\Delta$ inter-ring). An additional $\mathrm{CH}$ bending virbration of the methylene group $\left(\delta^{s} \mathrm{CH}_{2}\right)$ is of $a_{g}$ type in the case of fluorene. 
Table 1 Fundamental Raman wavenumbers $\left(\mathrm{cm}^{-1}\right)$ and assignments for the fluorene, dibenzofuran and dibenzothiophen triplet states $\left(T_{1}\right)$ and for the fluorene radical cation $\left(R^{+}\right)$, compared to the ground state $\left(S_{0}\right)$, in the $200-1800 \mathrm{~cm}^{-1}$ region

\begin{tabular}{|c|c|c|c|c|c|c|c|}
\hline \multirow{2}{*}{$\begin{array}{l}a_{g} \text { normal } \\
\text { modes }^{\mathrm{a}, \mathrm{b}}\end{array}$} & \multicolumn{3}{|c|}{ Fluorene } & \multicolumn{2}{|c|}{ Dibenzofuran } & \multicolumn{2}{|c|}{ Dibenzothiophen } \\
\hline & $S_{0}$ & $R^{+}$ & $T_{1}$ & $S_{0}$ & $T_{1}$ & $S_{0}$ & $T_{1}$ \\
\hline $8 a$ & 1,610 & 1,616 & 1,577 & 1,637 & 1,586 & 1,600 & 1,531 \\
\hline $8 \mathrm{~b}$ & 1,574 & 1,572 & 1,534 & 1,596 & 1,470 & 1,558 & - \\
\hline $19 a$ & 1,477 & 1,488 & 1,460 & 1,488 & 1,454 & 1,477 & 1,445 \\
\hline $19 b$ & 1,448 & 1,436 & - & 1,447 & 1,440 & - & - \\
\hline$\delta^{s} \mathrm{CH}_{2}$ & 1,397 & 1,415 & - & - & - & - & - \\
\hline 14 & 1,342 & - & - & 1,356 & - & 1,332 & - \\
\hline 3 & 1,328 & - & 1,310 & 1,347 & 1,320 & 1,319 & - \\
\hline$v$ inter-ring & 1,295 & 1,340 & 1,342 & 1,310 & 1,345 & 1,310 & 1,330 \\
\hline$v^{s}$ ring-R-ring & 1,231 & 1,182 & - & 1,247 & - & 419 & - \\
\hline $9 \mathrm{a}$ & 1,152 & 1,159 & 1,155 & 1,149 & $1,154^{\mathrm{c}}$ & 1,233 & - \\
\hline $18 \mathrm{a}$ & 1,092 & 1,109 & - & 1,102 & - & 1,133 & - \\
\hline $18 \mathrm{~b}$ & 1,019 & 1,005 & 965 & 1,012 & 964 & 1,027 & 977 \\
\hline 1 & 843 & 835 & 813 & 851 & 818 & 1,070 & $1,079^{c}$ \\
\hline 12 & 741 & 729 & 747 & 748 & - & 704 & - \\
\hline $6 \mathrm{~b}$ & 633 & 612 & - & 662 & - & - & - \\
\hline $6 a$ & 418 & 415 & 408 & 430 & - & 410 & - \\
\hline$\Delta$ inter-ring & 218 & - & - & 222 & - & 217 & - \\
\hline
\end{tabular}

$v=$ stretch, $\delta, \Delta=$ in-plane distortions.

b in the hypothesis of a planar structure with $C_{2 v}$ symmetry.

c very weak signals.

Table 2 Assignment of the Raman overtone and combination bands $\left(\mathrm{cm}^{-1}\right)$ for the fluorene radical cation $\left(R^{+\cdot}\right)$ and triplet state $\left(T_{1}\right)^{\mathrm{a}}$

\begin{tabular}{|c|c|c|c|}
\hline \multicolumn{2}{|l|}{$R^{+}$} & \multirow{2}{*}{$\frac{T_{1}}{370 \mathrm{~nm}}$} & \multirow[t]{2}{*}{ Assignment } \\
\hline Probe $370 \mathrm{~nm}$ & $625 \mathrm{~nm}$ & & \\
\hline 1,910 & - & - & $12+v$ ring-R-ring \\
\hline - & - & 1,868 & $19 a+6 a$ \\
\hline 1,839 & 1,840 & 1,778 & $18 b+1$ \\
\hline 1,794 & - & - & $6 \mathrm{~b}+v$ ring-R-ring \\
\hline 1,732 & - & - & $12+18 b$ \\
\hline$(1,565)$ & - & - & $12+1$ \\
\hline 1,675 & - & - & $2 \times 1$ \\
\hline \multirow[t]{2}{*}{1,459} & - & - & $2 \times 12$ \\
\hline & 1,419 & 1,374 & $18 b+6 a$ \\
\hline \multirow{2}{*}{$(1,340)$} & - & - & $12+6 b$ \\
\hline & 1,254 & 1,222 & $1+6 a$ \\
\hline 1,224 & - & - & $2 \times 6 b$ \\
\hline
\end{tabular}

a Wavenumbers in parentheses correspond to observed shoulders.

Strong couplings certainly take place between these vibrational groups and serious difficulties for comparing the ground and transient state vibrations may be expected if important variations in these vibrational couplings appear where modifying the electronic configuration.

However, it turns out from our previous analyses of transient Raman spectra ${ }^{6-11}$ 
that, in the case of large aromatic molecules, a correlation can be established between the ground state and the excited transient state vibrations. In fact, in polyatomic molecules having high $\pi$-density and strong electronic conjugation resonance ability, structural distortions in the transient states with respect to the ground state are largely distributed on several bonds in such a way that each bond is only moderately disturbed. Accordingly, in the assumption that the anharmonic couplings between the different $a_{g}$ vibrations (Dushinsky effect) are negligible, the same normal mode representation can be utilized in the different electronic states and we have adopted a unique notation for describing the vibrations in the $S_{0}, T_{1}$ and $R^{+}$. states. A detailed vibrational analysis of the title molecules and of several isotopic derivatives of fluorene (rings and/or methylene group hydrogenated/ deuterated) in the ground state $S_{0}{ }^{24}$ has indicated that, in these systems, the ring normal modes can be compared (frequency, isotopic effects) to the ring vibrations of ortho-disubstituted benzene molecules and thus can be described using the Wilson's notation for benzene, as adapted by Varsanyi for ortho-disubstituted benzenes. ${ }^{25}$ In this formalism, the thirteen $a_{g}$ ring vibrations of the fluorene-type molecules correspond to the symmetric combination (the two rings oscillate in phase) of the ortho-disubstituted benzene modes $8 \mathrm{a}, 8 \mathrm{~b}, 19 \mathrm{a}, 19 \mathrm{~b}, 14,1,12,6 \mathrm{a}, 6 \mathrm{~b}, 9 \mathrm{a}, 3,18 \mathrm{a}$ and $18 \mathrm{~b}$.

The assignments proposed in Table 1 for the transient state spectra are based on comparisons with the related ground state spectra and also with the assignments made previously for the biphenyl triplet state and radical cation species. ${ }^{10,11}$ As expected, most of the observed Raman bands are ascribed to totally symmetric vibrations in the assumption of planar, $C_{2 v}$ conformations. Nevertheless, several very weak signals in the radical cation spectrum of fluorene recorded at $625 \mathrm{~nm}$ remain unassigned (e.g. 1543, 1059, 1032, 970, 634 and $625 \mathrm{~cm}^{-1}$ ). They may be ascribed either to vibrations of another transient (photoproduct, dimer ion . . .), or to non-symmetric vibrations of the radical cation, the activity of which would reflect a slight distortion of the structure with respect to the $C_{2 v}$ symmetry.

\section{Radical Cation of Fluorene}

The shifts in frequency noted between the $S_{0}$ and $R^{+}$. spectra of fluorene are generally weak $\left(\Delta v<20 \mathrm{~cm}^{-1}\right)$. The only significant variations are observed for the two bands located at 1295 and $1231 \mathrm{~cm}^{-1}$ in the $S_{0}$ spectrum, which are shifted upon ionization by $+45 \mathrm{~cm}^{-1}$ and $-49 \mathrm{~cm}^{-1}$, respectively. According to our assignments, the first peak corresponds to a vibration involving a large contribution from the inter-ring stretching coordinate and the second one to a vibration predominantly characteristic of the ring- $\mathrm{CH}_{2}$-ring symmetric stretching coordinate. This suggests that the main structural distortion resulting from the $R^{+\cdot} \leftarrow S_{0}$ transition is a strengthening of the inter-ring $C C$ bond order and a simultaneous lengthening of the ring- $\mathrm{CH}_{2} \mathrm{CC}$ bonds, in accord with an increase of the quinoidal character of the structure (dashed trace in Scheme 1).

The increase of the inter-ring stretching frequency is similar to that found for the biphenyl radical cation $\left(\Delta v=+57 \mathrm{~cm}^{-1}\right),{ }^{11}$ indicating that fluorene and biphenyl 


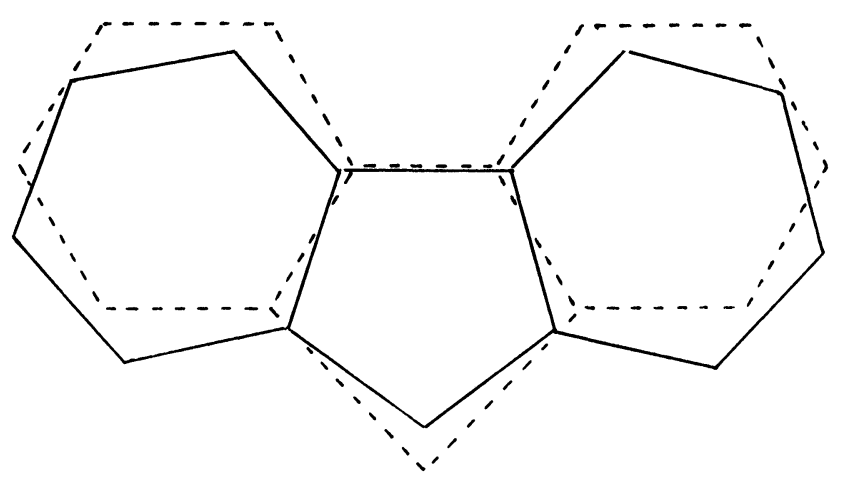

Scheme 1.

present comparable quinoidal distortions of the ring-ring skeleton upon ionization. This result confirms the resemblance of the electronic configurations of these two radical cations, in agreement with the predictions from simple MO considerations: in both compounds, ionization results from the ejection of an electron from the HOMO $\left(\phi_{6}\right)$ which has a marked anti-bonding character with respect to the inter-ring coordinate.

As can be seen in Figure 3, the spectrum obtained with excitation at $370 \mathrm{~nm}$ (trace B) is drastically different from that recorded upon $625 \mathrm{~nm}$ excitation (trace C). Striking variations in band intensity and enhancement effects are observed when changing the probe wavelength. As stated above, these variations in Raman intensity reflect the variations of vibronic activity (i.e., of Franck-Condon overlap) in the two electronic transitions $R^{+.^{*}} \leftarrow R^{+\cdot}$ which are in resonance, and are thus characteristic of the distortion of the molecular geometry in the related resonant states $R^{+. *}$. The interpretation of these intensity enhancement effects in terms of structural distortions appears much more difficult in this compound than in biphenyl in reason of the lower symmetry of the molecule and of the larger number of observed Raman bands. However, a very typical behavior is remarked for the two peaks ascribed to the ring vibrations $8 \mathrm{a}\left(1616 \mathrm{~cm}^{-1}\right)$ and $19 \mathrm{a}\left(1488 \mathrm{~cm}^{-1}\right)$. The first one is by far the most enhanced upon excitation at $370 \mathrm{~nm}$ whereas the second one is prominent on excitation at $625 \mathrm{~nm}$. Such a drastic inversion of intensity has been already encountered for biphenyl, ${ }^{11}$ indicating that the origins of the two electronic transitions $R^{+.^{*}} \leftarrow R^{+\cdot}$ in the UV and visible regions are comparable in fluorene and in biphenyl. Another common point between the two molecules is the similar intensity of the $v$ inter-ring band $\left(1340 \mathrm{~cm}^{-1}\right.$ in fluorene $\left.\mathrm{e}^{+}\right)$in the spectra excited at 370 and $625 \mathrm{~nm}$. In regard to these dominant resemblances, it seems reasonable to transpose to fluorene ${ }^{+\cdot}$ the schematic orbital diagram established for biphenyl ${ }^{+\cdot}$ (Figure 8 in ref. 11), which means that (i) the electron density distributions of the orbitals lying in the HOMO/LUMO region $\left(\phi_{3}-\phi_{10}\right)$ are not significantly altered in going from biphenyl to fluorene and (ii) the UV and visible absorptions of fluorene ${ }^{+\cdot}$ can be related to the $\phi_{7} \leftarrow \phi_{6}$ and $\phi_{6} \leftarrow \phi_{3}$ excitations of biphenyl ${ }^{+}$, respectively. 


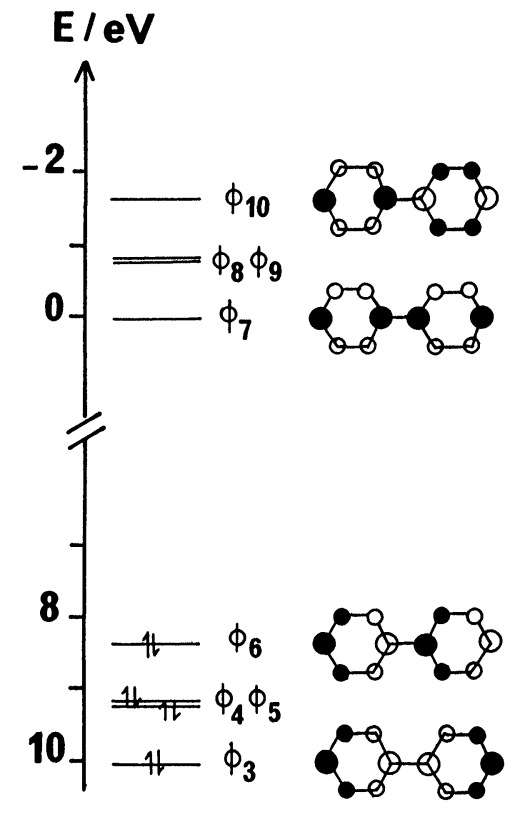

Figure 6 Molecular orbital diagram of biphenyl in the HOMO-LUMO region (from ref. 26) and schematic representation of some characteristic orbitals.

According to the schematic representation of the considered MOs (Figure 6), the $\phi_{7} \leftarrow \phi_{6}$ transition leads mainly to a reinforcement of the $\pi$-density on the inter-ring bond and on the $C_{2} C_{3}$ and $C_{5} C_{6}$ ring bonds, which is consistent with the resonance activity of modes 8a (see Figure 7) and $v$ inter-ring; and the $\phi_{6} \leftarrow \phi_{3}$ transition induces mainly a decrease in the inter-ring $\pi$-density and a distortion with respect to the inter-ring bond in the sense of the atomic displacements related to mode $19 \mathrm{a}$ (see Figure 7), in agreement with the resonance activity of these two vibrations.

In conclusion, the comparable resonance Raman behavior of modes $8 \mathrm{a}, 19 \mathrm{a}$ and $v$ inter-ring in the spectra of biphenyl ${ }^{+\cdot}$ and fluorene ${ }^{+\cdot}$. shows that the presence of the methylene group does not disturb markedly the electronic properties of the biphenyl system. The apparent confusion in the intensity enhancements noticed for the other vibrations can be accounted for by the lower symmetry and the larger mechanic constraints and couplings induced by this group in the fluorene framework.

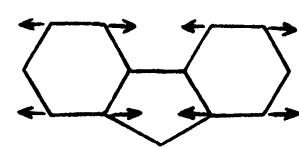

$8 \mathbf{a}$

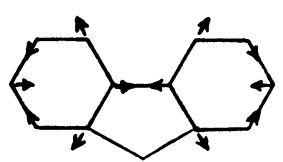

$19 \mathrm{a}$

Figure 7 Schematic representation of the cartesian displacements of modes $8 \mathrm{a}$ and $19 \mathrm{a}$. 


\section{Triplet State of Fluorene}

The localizaton of modes $8 \mathrm{a}, 8 \mathrm{~b}, 19 \mathrm{a}, 18 \mathrm{~b}, 1,12$ and $6 \mathrm{a}$ (see Table 1 ) is fairly reliable but the assignment of the bands lying in the $1300-1350 \mathrm{~cm}^{-1}$ region is only tentative. In this respect, our assignment differs from that proposed in ref. 20 for the CARS spectrum of $T_{1}$ fluorene. In particular, the authors ascribe the $1310 \mathrm{~cm}^{-1}$ signal (1312 $\mathrm{cm}^{-1}$ in the CARS spectrum) to a mode containing a main contribution from the $\delta^{s} \mathrm{CH}_{2}$ component, which is contradictory with the fact that this mode is still observed in the triplet Raman spectrum of dibenzofuran (see Table 1). On the other hand, they associate the $T_{1}$ signal at $1342 \mathrm{~cm}^{-1}\left(1337 \mathrm{~cm}^{-1}\right.$ in the CARS spectrum) to a ground state band at $1397 \mathrm{~cm}^{-1}$, which can be unambiguously ascribed to the $\delta^{s} \mathrm{CH}_{2}$ vibration in view of its isotopic behaviour. ${ }^{24}$ The observation of this $T_{1}$ signal in the Raman spectra of dibenzofuran $\left(1345 \mathrm{~cm}^{-1}\right)$ and dibenzothiophen $\left(1330 \mathrm{~cm}^{-1}\right)$ is also in contradiction with the assignment proposed in ref. 20.

As can be seen in Table 1, significant frequency decreases are noted for the ring modes $8 \mathrm{a}\left(-33 \mathrm{~cm}^{-1}\right), 8 \mathrm{~b}\left(-40 \mathrm{~cm}^{-1}\right), 19 \mathrm{a}\left(-17 \mathrm{~cm}^{-1}\right), 3\left(-18 \mathrm{~cm}^{-1}\right), 18 \mathrm{~b}$ $\left(-54 \mathrm{~cm}^{-1}\right), 1\left(-30 \mathrm{~cm}^{-1}\right)$ and $6 \mathrm{a}\left(-10 \mathrm{~cm}^{-1}\right)$ in going from $S_{0}$ to $T_{1}$ state. These negative shifts reflect a noticeable lowering of the $\pi$-bonding density in the rings, in agreement with the presence of an electron in a $\pi^{*}$ orbital. In contrast, according to the present assignment, the inter-ring stretching mode undergoes a $47 \mathrm{~cm}^{-1}$ positive shift which indicates that important strengthing of the inter-ring bond order takes place in the triplet state, as previously observed for $T_{1}$ biphenyl $(\Delta v=$ $\left.+79 \mathrm{~cm}^{-1}\right){ }^{10,11}$ Therefore, in both cases, a large contribution from a quinoidal-type conformation where the unpaired electrons are in para position may be assumed (Scheme 2).

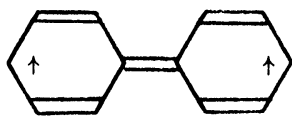

$\mathrm{T}_{1}$ bipheny 1

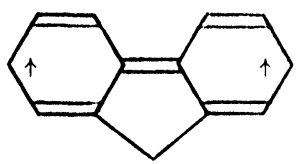

$\mathrm{T}_{1}$ fluorene

Scheme 2.

This result confirms the prediction from MO calculations ${ }^{18}$ that $T_{1}$ fluorene and $T_{1}$ biphenyl have comparable orbital natures and results from a LUMO $\left(\phi_{7}\right) \leftarrow \mathrm{HOMO}$ $\left(\phi_{6}\right) \pi \pi^{*}$ excitation.

Consider now the resonance Raman activity and relative band intensities. As observed for biphenyl, ${ }^{11}$ the triplet state spectrum of fluorene excited at $370 \mathrm{~nm}$ (Figure 3, trace A) is completely different from the radical cation spectrum recorded with the same wavelength (trace B) but it can be better compared to that excited at $625 \mathrm{~nm}$ (trace C). The main analogies are the high enhancement noted for mode 19a, the very weak intensity for mode 12 , and a similar activity for the combination bands (see Table 2). This indicates that comparable distortions of the $T_{1}$ and $\mathrm{R}^{+} \cdot$ structures 
take place in the corresponding resonant states $T_{n}$ and $R^{+. .^{*}}$, respectively. Therefore, in fluorene as in biphenyl the $T_{n} \leftarrow T_{1}$ transition at $370 \mathrm{~nm}$ is closely related to the $R^{+\cdot^{*}} \leftarrow R^{+\cdot}$ transition in the $600-700 \mathrm{~nm}$ range and is ascribable mainly to a $\phi_{6} \leftarrow \phi_{3}$ type excitation.

\section{Triplet State of Dibenzofuran and Dibenzothiophen}

The triplet state spectra of dibenzofuran and dibenzothiophen (Figure 4) have much lower signal-to-noise aspect than the spectrum of $T_{1}$ fluorene. In consequence, few Raman peaks can be detected for the heterocyclic molecules. However, the observed bands present similar frequency and intensity characteristics as the triplet bands of fluorene. One notes essentially a negative shift for the ring vibrations and a positive shift for the $v$ inter-ring mode on going from $S_{0}$ to $T_{1}$ states (see Table 1). Accordingly the distortion of the $T_{1}$ structures with respect to the ground state ones is comparable in dibenzofuran, dibenzothiphen and fluorene. This is in agreement with the conclusion from electron paramagnetic resonance measurements that replacing the methylene group by a heteroatom has no significant effect on the $T_{1} \leftarrow S_{0}$ transition. ${ }^{19}$ In all cases, the triplet state configuration results mainly from a LUMO $\leftarrow$ HOMO $\left(\pi \pi^{*}\right)$ excitation as in biphenyl.

Anyhow, the decrease in frequency noted for modes $8 \mathrm{a}$ and 19a on going from $S_{0}$ to $T_{1}$ state is more pronounced in dibenzofuran $\left(-51\right.$ and $-34 \mathrm{~cm}^{-1}$, respectively) and in dibenzothiophen ( -69 and $-32 \mathrm{~cm}^{-1}$, respectively) than in fluorene $(-33$ and $-17 \mathrm{~cm}^{-1}$, respectively, whereas the increase in the $v$ inter-ring frequency is rather attenuated in dibenzofuran $\left(+35 \mathrm{~cm}^{-1}\right)$ and dibenzothiophen $\left(+20 \mathrm{~cm}^{-1}\right)$ compared to fluorene $\left(+47 \mathrm{~cm}^{+1}\right)$. This suggests that the $\pi^{*}$ charge is more localized on the rings and that the $T_{1}$ structures present a less quinoidal character in the heterocyclic derivatives than in fluorene.

In conclusion, this preliminary work on fluorene-type compounds demonstrates that it is possible to obtain good-quality Raman spectra of the first triplet state and of the radical cation photochemical transients of fluorene in solution. At first appearance, a notable analogy is found between these spectra and those reported for biphenyl, ${ }^{10,11}$ in accord with previous results from electron paramagnetic resonance ${ }^{19}$ and with predictions from MO calculations. ${ }^{18}$ However, more detailed and reliable Raman assignments are necessary to support the present analysis and to provide more complete information on the transient structures. Further studies, which involve a series of isotopically substituted derivatives of fluorene, are thus currently in progress.

\section{References}

1. (a) M. Bridoux, C.R. Acad. Sci. 258, 5620 (1964); (b) M. Bridoux and M. Delaye, Nouv. Rev. Opt. Apl. 1, 23 (1970); (c) M. Delhaye, Lasers in Physical Chemistry and Biophysics (Elsevier, Amsterdam, $1985)$, p. 213.

2. (a) M. Bridoux and M. Delhaye, Advances in Infrared and Raman Spectroscopy (R. J. H. Clark and R. E. Hester, eds., Heyden, London, 1976), 2, 4, p. 140; (b) M. Bridoux, A. Deffontaine and C. Reiss, C.R. Acad. Sci. Ser. C, 282, 771 (1976); (c) M. Bridoux, A. Deffontaine, M. Delhaye and F. Grase, Il. Nuovo Climento 63, 91 (1981). 
3. (a) A. Deffontaine, A. Chapput, G. Buntinx and M. Bridoux, Spectros. Int. J. 2, 69 (1983); (b) A. Deffontaine, M. Bridoux, M. Delhaye, E. Da Silva and W. Hug, Rev. Phys. Appl. 19, 415 (1984).

4. R. Wilbrandt, N. H. Jensen, P. Pagsberg, A. H. Silleson and K. B. Hansen, Nature 276, 167 (1978).

5. (a) G. H. Atkinson, ed., Time-Resolved Vibrational Spectroscopy (Academic Press, New York, 1983); (b) A. Laubereau and M. Stockburger, eds. Time-Resolved Vibrational Spectroscopy (Springer, Berlin, 1985); (c) H. Hamaguchi, Vibrational Spectra and Structure (J. R. Durig, ed., Elsevier, Amsterdam, 1987), 2, 4, p. 270.

6. O. Poizat, A. Bourkba, G. Buntinx, A. Deffontaine and M. Bridoux, J. Chem. Phys. 87, 6379 (1987).

7. O. Poizat, V. Guichard and G. Buntinx, J. Chem. Phys. 90, 4697 (1989).

8. V. Guichard, A. Bourkba, O. Poizat and G. Buntinx, J. Phys. Chem. 93, 4429 (1989).

9. V. Guichard, O. Poizat and G. Buntinx, J. Phys. Chem. 93, 4436 (1989).

10. G. Buntinx, A. Benbouazza, O. Poizat and V. Guichard, Chem. Phys. Letters 153, 279 (1988).

11. G. Buntinx and O. Poizat, J. Chem. Phys. 91, 2153 (1989).

12. M. F. Alfimov, I. G. Batekha, Y. Sheck and V. I. Gerko, Spectrochim. Acta 27A, 329 (1971).

13. V. A. Godik, S. N. Davydov, G. G. Konoplev, A. N. Rodionov and D. N. Shigorin, Theor. Eksp. Khim. (Engl. Transl.) 18, 323 (1982).

14. V. A. Godik, S. N. Davydov, A. N. Rodionov and D. N. Shigorin, Zh. Fiz. Khim. (Engl. Transl.) 59, 1733 (1985).

15. T. G. Pavlopoulos, Spectrochim. Acta 42A, 1307 (1986).

16. M. O. Delcourt and M. J. Rossi, J. Phys. Chem. 86, 3233 (1982).

17. O. M. Andreev, V. A. Smirnov and M. V. Alfimov, J. Photochem. 7, 149 (1977).

18. B. Dick and G. Hohlneicker, Chem. Phys. 94, 131 (1985).

19. S. Siegel and H. S. Judeikis, J. Phys. Chem. 70, 2201 (1966).

20. S. Matsunuma, T. Kamisuki, Y. Adachi, S. Maeda and C. Hirose, Spectrochim. Acta 44A, 1403 (1988).

21. I. B. Berlman, Handbook of Fluorescence Spectra of Aromatic Molecules (Academic Press, New York, 1971).

22. D. Lavalette and C. Treteau, Chem. Phys. Letters 29, 204 (1968).

23. A. C. Albrecht, J. Chem. Phys. 34, 1476 (1961).

24. O. Poizat and G. Buntinx, in preparation.

25. G. Varsanyi, Assignments for Vibrational Spectra of Benzene Derivatives (I. Land, ed., Adam Hilger, London, 1974) vol. 1.

26. M. J. S. Dewar and N. Trinajstic, Czech. Chem. Commun. 35, 3136 (1970). 